\title{
DESIGN AND FABRICATION OF A DISCOMFORT INDEX METER FOR DETERMINATION OF STRESS IN LIVESTOCK
}

\author{
F. R. Falayi $1^{*}$ and M. O. Olanipekun ${ }^{2}$ \\ 1,2 Dept. of Agricultural and EnVironmental Engr, Fed. Univ. of Technology, AKure Ondo State NigERIA \\ E-mail addresses:1frfalayi@futa.edu, 2olanipekunmutiu@gmail.com.
}

\begin{abstract}
This research was aimed at designing and developing a discomfort index meter for evaluating transportation stress on broilers. The newly developed discomfort index meter is capable of recording and logging data every 5 seconds. The device was designed using LM35 electronic sensor to measure the dry bulb and wet bulb temperatures in degree Celsius $\left({ }^{\circ} \mathrm{C}\right)$ and then use it to compute the discomfort level using the Thom model with the help of microcontroller. The newly developed equipment was successfully used to evaluate the effect of transport on the level of stress of four weeks old broilers over various distances and stocking densities using different means of transportation. The equipment was calibrated and compared with other available measuring instruments which showed that a high correlation exist between the values $\left(r^{2}=0.89\right.$ ).Also, a single factor (one-way) analysis of variance (ANOVA) was carried out and the results showed no significant difference between the two sets of values at 0.05 level of confidence which implies that the two readings are identical. The relative humidity readings of the device were compared with that of standard digital hygrometer reading using a regression analysis which also showed a high correlation coefficient $\left(r^{2}=0.98\right)$. A straight line regression equation $y=0.9497 x+5.1542$ describes the relationship. The equipment is affordable and therefore recommended for determining stress on broilers and other livestock animals in transit.
\end{abstract}

Keywords: Discomfort meter, relative humidity, Temperature, Microcontroller, hygrometer

\section{INTRODUCTION}

Poultry birds are exposed to a number of concurrent stressors during transportation. Mitchell and Kettle well [1] observed that thermal challenges (both elevated thermal loads and cold stress) constitute the main threat to the birds' well-being and survival. They reported multiple factors during transportation that can create negative effects on livestock. Among these factors are: changes in temperature and humidity, airflow and gas, the density of the cargo and road conditions, the design of the truck and driver performance, these related to the greater or lesser intensity of mechanical vibrations. Daghir [2] stated that the effects of thermal stress can be worsened by withdrawal of food or water and by exposure to vibrations and accelerations. Existing pathologies and injuries may further compound the situation [3]. Whilst genetic selection in broiler chickens has resulted in major improvements in growth rates and production efficiency, these advances may also be associated with a reduced resistance to thermal stress, altered heat exchange capacity and muscle and cardiovascular pathologies. These pathological and physiological conditions may render the birds more susceptible to thermal stress and may form a part of the "transportation stress" experienced by poultry birds. These conditions can also lead to increased mortalities during exposure to hostile thermal microenvironments in transit and contribute to the increase in transport mortalities observed during challenging meteorological conditions. Solutions to these problems may be provided by changes in transportation practices and procedures, improvements in the design of transport containers, vehicles and their ventilation systems and the introduction of genetic selection programmes for more robust lines that are less susceptible to thermal stress, exhibit fewer, idiopathic pathologies, and/or simply grow more slowly. Welfare during transport may be improved by a more holistic consideration of the birds' physiology, rearing conditions, pre-transport handling and the prevailing conditions and stressors that may be imposed during the journey.

All modes of transport involve the placement of birds or chicks into transport containers which are subsequently loaded on to vehicles, aircraft or vessels for translocation to their intermediate or final destinations. The procedures and practices involved in transportation and

\footnotetext{
* Corresponding author, tel: + 234-803-394- 4486
} 
the micro-environments prevailing in containers and vehicles may impose varying degrees of stress upon the birds which will affect their welfare status, health and productive efficiency depending upon the magnitude of the challenges imposed. Nicol and Scott [4] stated that in transit, birds may be exposed to a variety of potential stressors including the thermal demands of the transport micro-environment, acceleration, vibration, motion, impacts, fasting, and withdrawal of water, social disruption and noise. Each of these factors and their various combinations may impose stress upon the birds, but it is well recognized that thermal challenges and in particular heat stress constitute the major threat to animal well-being and productivity.

High mortality rate is always recorded for poultry birds during transportation in Nigeria and other humid tropical regions of the world in general. Little or no research efforts have been made on the measurement of stressor indexes with the aim of reducing stresses in livestock animals in transit. Hence the aim of this study is to develop a discomfort meter capable of measuring, displaying and logging data of the discomfort level of poultry birds in transit.

\section{MATERIALS AND METHODS}

\subsection{Description of the Discomfort Index Meter}

Figure 1 shows the Relative Humidity and discomfort Index Meter. The outer components consist of wet and dry bulb thermometer, digital display, RH detector and memory card slot. The newly developed digital equipment is capable of logging data every 5 seconds. The meter can also measure the dry bulb and wet bulb temperatures in degree Celsius $\left({ }^{\circ} \mathrm{C}\right)$. The relative humidity $(\varnothing)$ of air-water mixture is defined as the ratio of the partial pressure of water vapour $\left(\mathrm{H}_{2} \mathrm{O}\right)$ in the mixture to the saturated vapour pressure of water at a given temperature.

The relative humidity is normally expressed as percentage and is calculated by using the following equation.

$$
\varnothing=\frac{e_{w} \times 100}{e^{*}{ }_{w}}
$$

In (1), $\varnothing$ is the relative humidity, $e_{w}$ is the partial pressure of water vapour and $e^{*}{ }_{w}$ is the Saturated vapour pressure

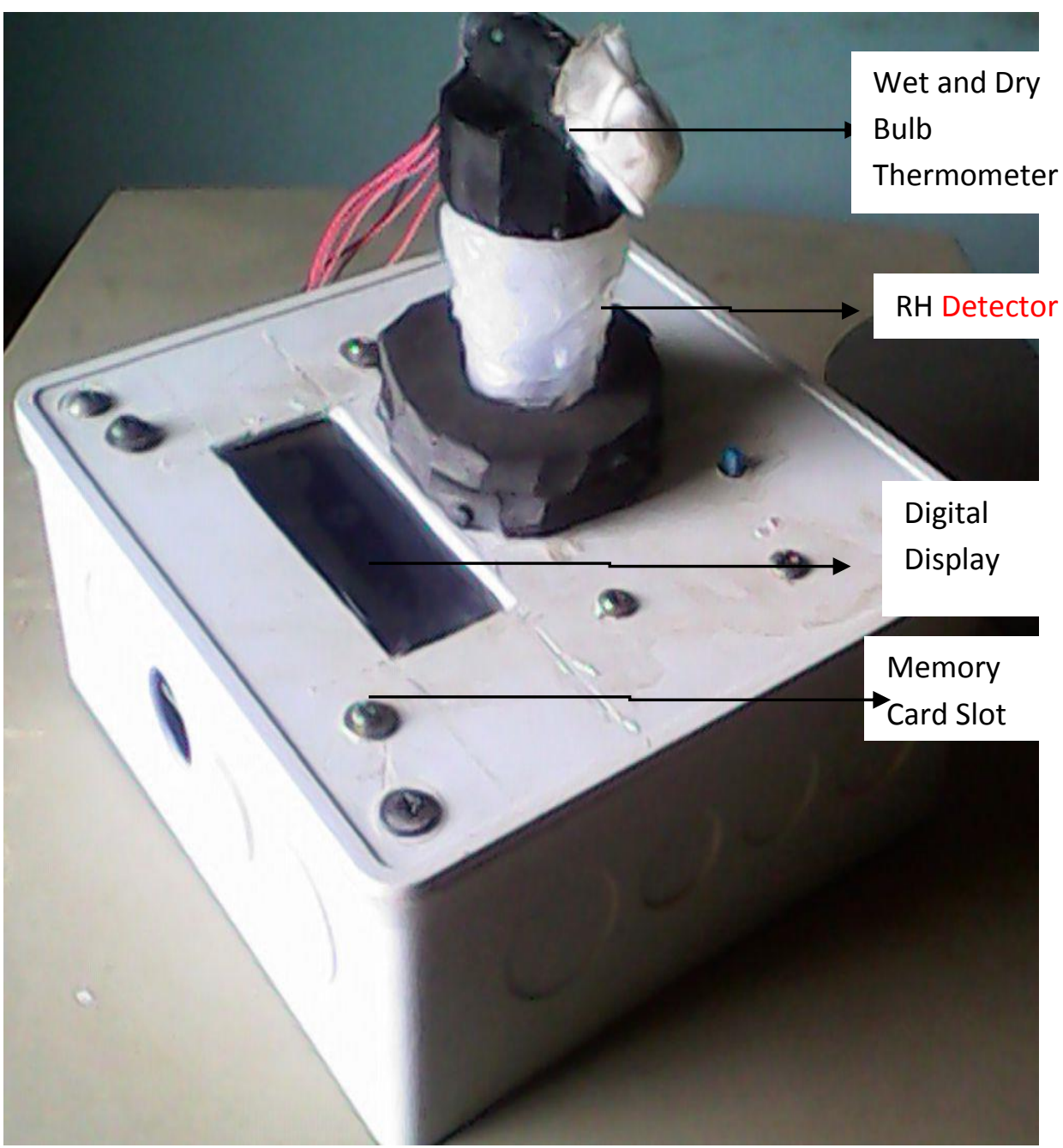

Figure: 1 Relative Humidity and discomfort Index Meter 


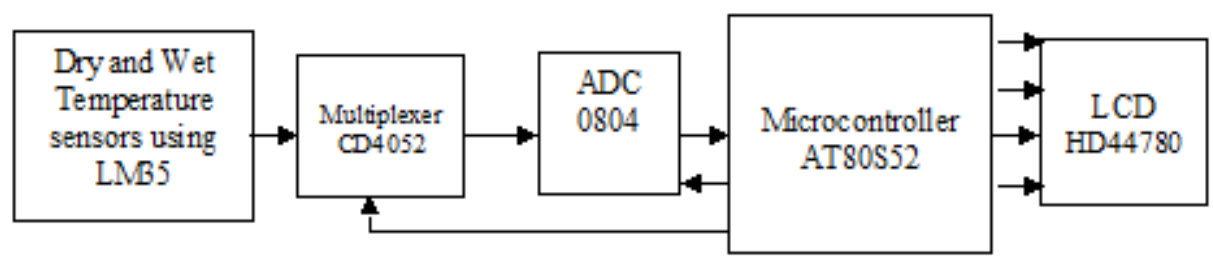

Figure 2: The Block Diagram of Discomfort Index Measurement Instrumentation System

\subsection{Design of Discomfort Index Meter}

Discomfort Index Meter consists of the following components namely dry and wet temperature sensors using LM35, multiplexer (CD4052), analog-to-digital converter (ADC0804), microcontroller (AT80S52) and intelligent display unit (LCD HD44780) as shown in figure 2 . These components were connected together to make up the system. Also, an assembly language was written to read dry and wet temperatures, and internally compute the value of discomforted index.

\subsection{Temperature Sensor Design}

The LM35Z or LM35 series are precision integratedcircuit temperature sensors, whose output voltage is linearly proportional to the Celsius (Centigrade) temperature. The simple connection of LM 35 at reference voltage Vs is for the range of temperature from $+2{ }^{\circ} \mathrm{C}$ to $150{ }^{\circ} \mathrm{C}$. Advantage of using LM35 is that it does not require external component for calibration or trimming. It has accuracies of $\pm 0.5{ }^{\circ} \mathrm{C}$ at room temperature and $\pm 0.75^{\circ} \mathrm{C}$ over full temperature range of sensor. The sensor sensitivity is $+10 \mathrm{mV} /{ }^{\circ} \mathrm{C}$. The LM35's low output impedance, linear output, and precise inherent calibration make readout and control circuitry easy [5]. It required single power supply of voltage range from $4 \mathrm{~V}$ to $30 \mathrm{~V}$. Its energy saving since it draws only 60 $\mu \mathrm{A}$ from its supply, it has very low self-heating, less than $0.1{ }^{\circ} \mathrm{C}$ in still air and with low impedance output, $0.1 \Omega$ for $1 \mathrm{~mA}$ load

\subsection{Wet Junction Construction}

The wick of the wet junction is made by passing three lengths of cotton sewing machine thread over the LM35Z junction or neck and binding them, not too tightly, with another length of the same thread. The six ends of the threads, which pass over the junction is glued with araldite while the two lengths of the binding thread form the wick [6].

\subsection{Circuit Description and its Operation}

Figure 3 shows the Complete Schematic Circuit for Discomfort. The level of discomfort index was determined using Thom model expressed as;

$$
\mathrm{DI}=0.4(\mathrm{Twd}+\mathrm{Twb})+4.8
$$

In (2) Twd, Twb, are the dry and wet bulb temperatures respectively.

The dry and wet thermometer was designed using microcontroller and Thom model equation was embedded inside the microcontroller. The system uses dry and wet temperature developed using semiconductor temperature sensors (LM35).Other materials required in the system are multiplexer (CD4052), analog to digital converter (ADC0804), microcontroller (AT80S52) and intelligent display unit (Hitachi's LCD HD44780). An assembly language was written to take in data and compute for the Thom model. The temperature measurement using LM35 for dry and wet temperatures was in agreement with convention built using mercury in glass thermometer [7].

According to Ewetumo [8], the temperature and humidity conditions, which invariably determine the values of comfort or discomfort, are graduated as shown in Table 1. The ideal temperature-humidity index for different ages of broilers is shown in Table 2 as stated by Silva [9]

Table 1 Categories of the Discomfort index

\begin{tabular}{|c|c|}
\hline $\begin{array}{l}\text { Level of discomfort } \\
\text { Index }\end{array}$ & Condition of room \\
\hline $\mathrm{DI}<21$ & Comfort for man \\
\hline $21<\mathrm{DI}<24$ & $\begin{array}{l}\text { The } 10 \% \text { of the total population } \\
\text { feels discomfort }\end{array}$ \\
\hline $24<\mathrm{DI}<26$ & $\begin{array}{l}\text { The } 50 \% \text { of the total population } \\
\text { feels discomfort }\end{array}$ \\
\hline $\mathrm{DI}>26$ & $\begin{array}{l}\text { The } 100 \% \text { of the total population } \\
\text { feels discomfort }\end{array}$ \\
\hline
\end{tabular}

Table 2: Ideal Temperature-Humidity index for different ages of broilers

\begin{tabular}{cccc}
\hline $\begin{array}{c}\text { Age } \\
\text { (weels) }\end{array}$ & Temperature $\left({ }^{\circ} \mathrm{C}\right)$ & $\begin{array}{c}\text { Humidity } \\
(\%)\end{array}$ & $\begin{array}{c}\text { THI } \\
\text { ideal }\end{array}$ \\
\hline 1 & $32-35$ & $60-70$ & $72.4-80$ \\
2 & $29-32$ & $60-70$ & $68.4-76$ \\
3 & $26-29$ & $60-70$ & $64.5-72$ \\
4 & $23-26$ & $60-70$ & $60.5-68$ \\
5 & $20-23$ & $60-70$ & $56.6-64$ \\
6 & 20 & $60-70$ & $56.6-60$ \\
8 & 20 & $60-70$ & $56.6-60$ \\
\hline \multicolumn{4}{c}{ Source: [9] } \\
\end{tabular}




\subsection{Criteria for choosing a Microcontroller}

Zhang [10] basic criteria was used in choosing the microcontroller suitable for the application. In analysing the needs of a microcontroller-based project, it is seen whether an 8- bit, 16-bit or 32-bit microcontroller can best handle the computing needs of the task most effectively. Among the other considerations in this category are:

(a) Speed: The highest speed that the microcontroller supports.

(b) Packaging: It may be a 40-pin DIP (dual inline package) or a QFP (quad flat package), or some other packaging format. This is important in terms of space, assembling, and prototyping the end product

(c) Power consumption: This is especially critical for battery-powered products.

(d) The number of I/O pins and the timer on the chip.

(e) How easy it is to upgrade to higher-performance or lower consumption versions.

(f) Costperunit: This is important in terms of the final cost of the product in which a microcontroller is used.

\subsection{Basic Description of Microcontroller AT89S52}

The 8051 family of microcontrollers is based on an architecture which is highly optimized for embedded control systems. It is used in a wide variety of applications from military equipment to automobiles to the keyboard. Manufacturers have added numerous features and peripherals to the 8051 such as I2C interfaces, analog to digital converters, watchdog timers, and pulse width modulated outputs. Variations of the 8051 with clock speeds up to $40 \mathrm{MHz}$ and voltage requirements down to 1.5 volts are available. This wide range of parts based on one core makes the 8051 family an excellent choice as the base architecture for a company's entire line of products since it can perform many functions and developers will only have to learn this on one platform.

The AT89S52 is a low-power, high-performance CMOS 8bit microcontroller with $8 \mathrm{~K}$ bytes of in-system programmable Flash memory. The device is manufactured using Atmel's high-density non-volatile memory technology and is compatible with the industrystandard 80C51 instruction set and pin out. The on-chip Flash allows the program memory to be reprogrammed in-system or by a conventional non-volatile memory programmer. By combining a versatile 8-bit CPU with insystem programmable Flash on a monolithic chip, the Atmel AT89S52 is a powerful microcontroller which provides a highly-flexible and cost-effective solution to many embedded control applications. In addition, the AT89S52 is designed with static logic for operation down to zero frequency and supports two software selectable power saving modes. The Idle Mode stops the CPU while allowing the RAM, timer/counters, serial port, and interrupt system to continue functioning. The Powerdown mode saves the RAM con-tents but freezes the oscillator, disabling all other chip functions until the next interrupt or hardware reset. The microcontroller is interfaced with the ADC with multiplexer inputs and call for the LCD mode display.

\subsection{Sensor Selector Unit}

Since two input signal sources are available CD4052 differential 4 channel multiplexer IC was used to select input one by one and is addressed by microcontroller and output signal of the multiplexer fed into the input of ADC0804, the output from ADC is sent to port 3 (P3) of AT89S52 controller. It samples the data every $1 \mathrm{~s}$.

\subsection{Analog-to-digital Conversion (ADC0804)}

Recommendations made by Pooja and Kapil [10] were used in the conversion. The ADC0804 is compatible with microprocessors. It is a 20-pin IC that works with $5 \mathrm{~V}$ supply. It converts the analogue input voltage to 8-bit digital output. The data bus is tri-state buffered. With eight bits, the resolution is $5 \mathrm{~V} / 255=19.6 \mathrm{mV}$. The inbuilt clock generator circuit produces a frequency of about $640 \mathrm{kHz}$ with R1 $=10$ kilo-ohms and $\mathrm{C} 4=150 \mathrm{pF}$, which connected timing components. The conversion time obtained is approximately 100 micro-second. The functions of other pins are given below:

Pin 1 (CS): The active low chip-select pin.

Pin 2 (RD): This active-low pin enables the digital output buffers. When high, the 8-bit bus will be in Hi-Z state.

Pin 3 (WR): This active-low pin is used to start the conversion.

Pin 9 (Vref/2): This is optional input pin. It is used only when the input signal range is small. When pin 9 is at $2 \mathrm{~V}$, the range is $0-4.0 \mathrm{~V}$, that is, twice the voltages applied to these pins. The analogue input can range from 0 to $5 \mathrm{~V}$. Pin $6(\mathrm{~V}+)$,

Pin 7 (V-): The actual input is the difference in voltages applied to these pins. The analogue input can range from $0-5 \mathrm{~V}$.

Pin 5 (INTR): This is an active-LOW interrupt, or halt pin. Pins 11-18

(DB7-DB0): These are the three-state buffered digital outputs.

Pin 8 (A GND): This is ground connection for the input analog voltage.

Pin 10 (D GND): This is ground for the device and digital outputs.

\subsection{Intelligent Liquid Crystal Display Unit}

The method used was similar to that of Yongsang and Minkyu [12].The LCD HD44780 standard requires 3 
control lines as well as either 4 or $8 \mathrm{I} / 0$ lines for the data bus. The user may select whether the LCD is to operate with a 4-bit data bus or an 8-bit data bus. If a 4-bit data bus is used the LCD will require a total of 7 data lines ( 3 control lines plus the 4 lines for the data bus). If an 8-bit data bus is used the LCD will require a total of 11 data lines ( 3 control lines plus the 8 lines for the data bus). The three control lines are referred to as EN, RS, and RW. The EN line is called "Enable." This control line is used to tell the LCD that you are sending data to it. To send data to the LCD, the program should make sure this line is low (0) and then set the other two control lines and/or put data on the data bus. When the other lines are completely ready, bring EN high (1) and wait for the minimum amount of time required by the LCD datasheet (this varies from LCD to LCD), and end by bringing it low (0) again. The RS line is the "Register Select" line. When RS is low (0), the data is to be treated as a command or special instruction (such as clear screen, position cursor, etc.). When RS is high (1), the data being sent is text data which should be displayed on the screen. To display any letter or digit on the screen you would set RS high. The RW line is the "Read/Write" control line. When RW is low (0), the information on the data bus is being written to the LCD. When RW is high (1), the program is effectively querying (or reading) the LCD. Only one instruction ("Get LCD status") is a read command. All others are write commands, so RW will almost always be low. Finally, the data bus consists of 4 or 8 lines (depending on the mode of operation selected by the user). In this study an 8-bit data bus is used, the lines are referred to as DB0, DB1, DB2, DB3, DB4, DB5, DB6, and DB7. Before intelligent liquid crystal display can be ready for use, it must be initialized and configured. The first instruction to send must tell the LCD whether the communication will be an 8-bit or 4-bit data bus.

\section{RESULTS AND DISCUSSION}

The equipment was evaluated by using it to measure the discomfort level of poultry birds in transit. Parameters measured included the dry bulb temperature, wet bulb temperature, relative humidity and the discomfort level. The readings from the equipment were compared with readings taken by other standard equipment such as digital thermometer and digital hygrometer.

The results of a regression analysis carried out for values of dry bulb temperature measured by the device and other digital thermometer(Range: 200/1370 ${ }^{\circ}$ CAccuracy: $\pm 0.2^{\circ} \mathrm{CModel}$ ACCD710P)is shown in Fig 4. The results show that a high correlation exists between the values $\left(r^{2}=0.89\right)$. Also, a single factor (one-way) analysis of variance (ANOVA) was carried out and the results is as shown in Table 3. From the results, there is no significant difference between the two sets of values at 0.05 level of confidence which implies that the two readings are identical.

The relative humidity readings of the device was compared with that of standard digital hygrometer reading, a regression analysis was carried out and the correlation is high $\left(\mathrm{r}^{2}=0.98\right)$ as shown in Figure 5 . A straight line regression equation $\mathrm{y}=0.9497 \mathrm{x}+5.1542$ describes the relationship.

This equipment is as good as others for practical applications such as measuring relative humidity and temperature of any microenvironment. For example, Ewetumo [8] used similar equipment for measurement of environmental parameters. Furthermore the empirical formula which forms the basis of the equipment has been based upon the feeling of comfort or discomfort, of a great number of people, with similar adaptive reactions to the environmental conditions [13].

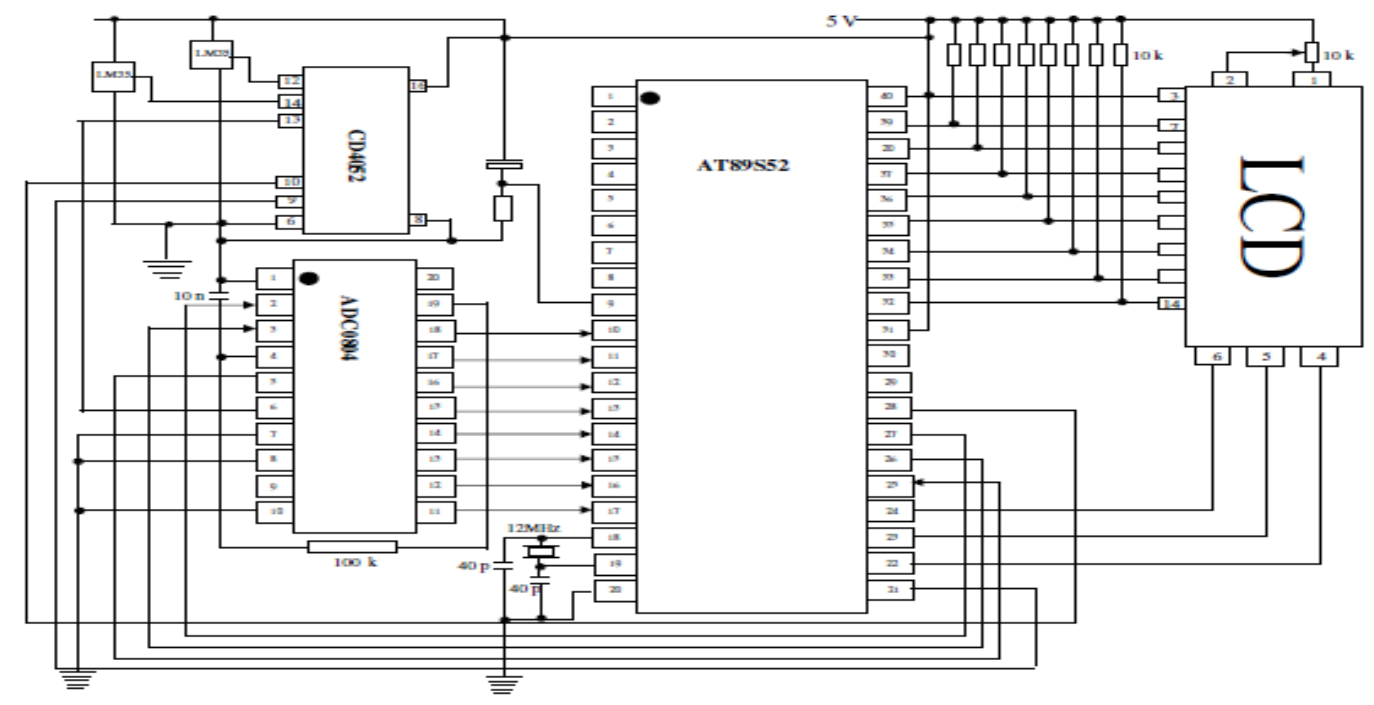

Figure 3: Complete Schematic Circuit for Discomfort 


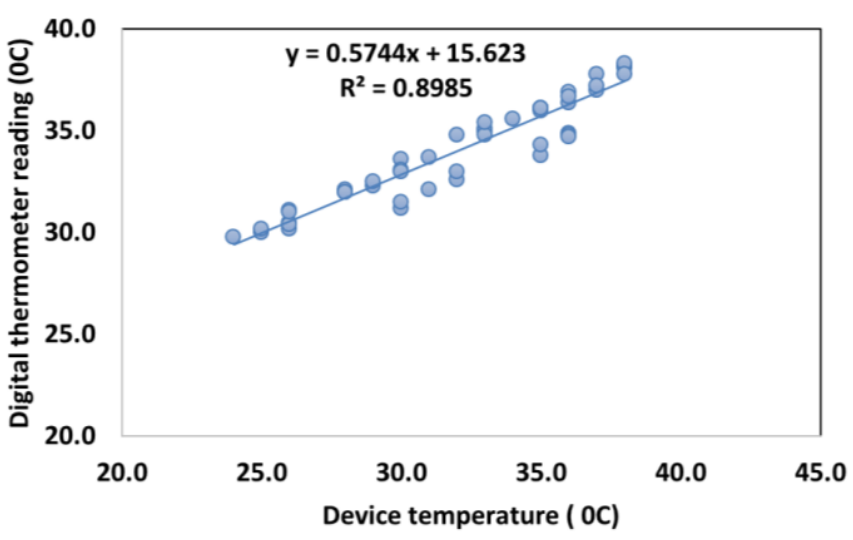

Figure 4: Calibration of device temperature readings

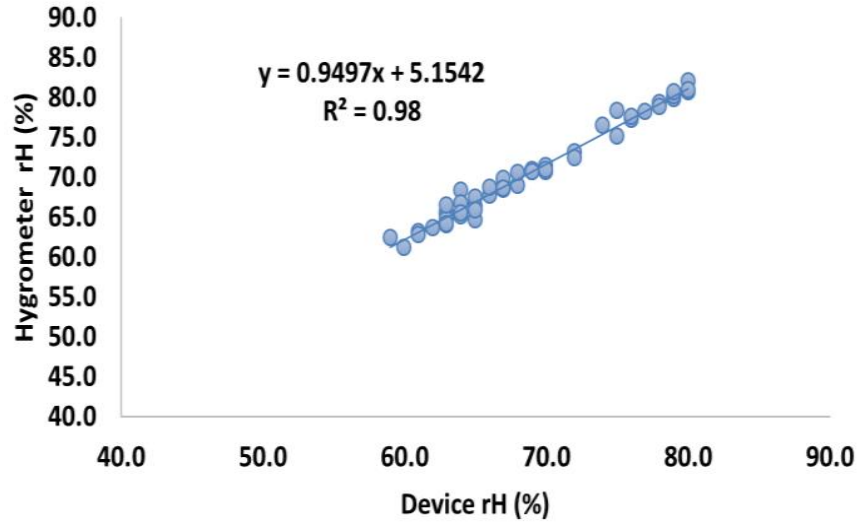

Figure 5: Calibration of device relative humidity readings

Table 3: Single Factor ANOVA

\begin{tabular}{|c|c|c|c|c|c|c|}
\hline \multicolumn{7}{|l|}{ SUMMARY } \\
\hline Groups & Count & Sum & Average & Variance & & \\
\hline Device & 50 & 3458 & 69.16 & 39.97388 & & \\
\hline Thermometer & 50 & 3541.8 & 70.836 & 36.79051 & & \\
\hline \multicolumn{7}{|l|}{ ANOVA } \\
\hline Source of Variation & $S S$ & $d f$ & $M S$ & $F$ & $P$-value & F crit \\
\hline Between Groups & 70.2244 & 1 & 70.2244 & 1.829609 & 0.179286 & 3.938111 \\
\hline Within Groups & 3761.455 & 98 & 38.3822 & & & \\
\hline Total & 3831.68 & 99 & & & & \\
\hline
\end{tabular}

\section{CONCLUSION}

Discomfort index meter was designed and developed for evaluating stress on poultry broilers and other livestock animals. The device was designed using LM35 to measure the dry bulb temperature and wet bulb temperatures in degree Celsius $\left({ }^{\circ} \mathrm{C}\right)$ and then use it to compute the discomfort level using the Thom model with the help of microcontroller. The equipment was calibrated and compared with other available measuring instruments which showed that a high correlation exist between the values $\left(r^{2}=0.89\right)$. Also, the results a single factor (one-way) analysis of variance (ANOVA) showed no significant difference between the two sets of values at 0.05 level of confidence which implies that the two readings are identical. The relative humidity readings of the device were compared with that of standard digital hygrometer readings using a regression analysis which also showed a high correlation coefficient $\left(\mathrm{r}^{2}=0.98\right)$. A straight line regression equation $\mathrm{y}=0.9497 \mathrm{x}+5.1542$ describes the relationship.

With this equipment at hand, the well-being and discomfort of livestock animals can easily be measured. The discomfort index meter is therefore considered appropriate for determining and evaluating stress imposed on birds either in the poultry house or during transportation.

\section{REFERENCES}

[1] Mitchell M. A. and P. J. Kettlewell Welfare of poultry during transport - a review. SAC, Sustainable Livestock Science, Sir Stephen Watson Building, Bush Estate, Penicuik, Midlothian, EH26 0PH, Scotland, UK.Pg 2-11, 2009.

[2] Daghir, N. J. Poultry production in hot climate, $2^{\text {nd }}$ Edition, Published by CAB International, Wallinford, Oxford, Oxfordshire, Uk, pp. 387, 2008.

[3] Dozier W. A., Thaxton J. P, Braton S.L., Morgan G.W., Miles D.M., Roush W.B., Lott B.D., Vizzier-Thaxton Y.. Stocking density on growth performance and processing yield of heavy broilers. Poultry science, 84: 1332 - 1338, 2005.

[4] Nicol, C.

J. and Scott, G. B. Pre-slaughter handling and transport of broiler chickens. Applied Animal Behaviour.Science. 28: 57-73 1990.

[5] Theophilus W. and Bhudi S. "A Microcontrollerbased Room Temperature Monitoring System", International Journal of Computer. Applications. Volume 53- No.1. . 0975 - 8887

[6] Xing Guo Quan "Temperature Characteristics and Application of LM35 Sensor", Biomedical Engineering Department of Xianmng College 
Xianning Hubei-437100 China, Article id 10077510(2007)11-0049-02,2007.

[7] Gao Mei Zhen "Principle and application of the LM 35 series temperature sensor", Hubei normal university Article Id:1671-1041(2005)01-0114-0 , 2005.

[8] Ewetumo T. Development of Digital Display Dry and Wet Thermometer International Journal of Physical Sciences, Nigeria. Vol. 2, (1):62-67,2007.

[9] Silva, ET. Index of temperature and humidity (THI) in poultry production for misprision of northwestern and pioneer north of Paraná. Journal of Applied Chemistry, 5 (4), pp. 385-390, 2007.

[10] Zhang Qian "Design of digital thermometer based on AT89C52 Single Chip Microcontroller",
International Conference on Electrical and Control Engineering (ICECE), China. 2010..

[11] Pooja S. and Kapil S. "Exploring the Serial Capabilities for 16x2LCD Interface", International Journal of Emerging Technology and Advanced Engineering, ISSN 2250-2459, Volume 2, Issue 11. 2012.

[12] Yongsang Y and Minkyu S. "Design of a 1.8V Lobit JOOMSPSCMOS Digital - to - Analog Converter with a Novel Delitchings Circuit and Inverse Thermometer Decoder", Asia-Pacific Conference on Circuits and Systems (APCCAS' 02), Volume-2, 2002.

[13] Metaxas, D. Bio meteorological indices and acclimatization in Greece during the summer. Technical Note No. 6, 321-332. 1970. 